\title{
L'ANALYSE DES CORRESPONDANCES DÉCENTRÉE : APPLICATION AUX PEUPLEMENTS ICHTYOLOGIQUES DU HAUT-RHÔNE.
}

\author{
S. DOLÉDEC, D. CHESSEL, J.M. OLIVIER
}

U.R.A. C.N.R.S. 1974, Ecologie des Eaux Douces et des Grands Fleuves, Université Lyon I, 43 Bd du 11 novembre 1918, 69622 Villeurbanne Cedex, France.

\section{RÉSUMÉ}

L'article décrit une nouvelle méthode d'ordination linéaire appelée Analyse Factorielle des Correspondances Décentrée (AFCD) qui fait partie de la famille des Analyses Factorielles des Correspondances sur modèles (ESCOUFIER, 1985). Cette méthode permet de prendre en compte l'hétérogénéité de l'effort d'échantillonnage des peuplements piscicoles par pêche électrique en grand fleuve. L'analyse de la distribution de huit espèces de poissons recensées à l'occasion de 32 campagnes de pêche, réalisées de 1978 à 1987 sur le Haut-Rhône français, a permis de tester les potentialités de l'AFCD par rapport à l'AFC classique. On montre que cette méthode est particulièrement adaptée à l'analyse de la structure de données ichtyologiques résultant d'échantillonnages ponctuels d'abondance. Elle permet en particulier pour les huit espèces considérées de séparer les profils à long terme du profil qu'aurait une espèce uniformément répartie entre les campagnes de pêches. L'ambiguitté d'interprétation liée au point de référence pris en AFC classique est ainsi levée.

Mots-clés : échantillonnage ponctuel, grand fleuve, peuplements piscicoles, Analyse Factorielle des Correspondances Décentrée.

\section{DECENTRED CORRESPONDENCE ANALYSIS APPLIED TO THE FISH COMMUNITIES OF THE UPPER RHÔNE RIVER.}

\begin{abstract}
The paper describes a new multivariate method called decentred correspondence analysis, which belongs to the correspondence analysis on models group (ESCOUFIER, 1985). This method enables users to take into account the heterogeneity of sampling effort of fish communities assessed by electric fishing in large rivers. The decentred correspondence analysis of the abundance distribution of eight fish species collected at 32 occasions, on the French Upper Rhône from 1978 to 1987 , demonstrates that it is a convenient alternative for analysing the structure of ichtyofauna collected with the point abundance sampling method. In particular, the species profiles may be separated by their distance to an expected species uniformely distributed among sampling occasions. As a result, the decentred correspondence analysis permits to circumvent the problem associated with the reference point implicitly selected in the classical correspondence analysis.
\end{abstract}

Key-words : point sampling, large river, fish communities, decentred correspondence analysis. 


\section{INTRODUCTION}

L'Analyse Factorielle des Correspondances (AFC) est une méthode multivariée très utilisée pour la description des structures faunistiques (tableau relevés-espèces). Une des premières utilisations en hydrobiologie est due à LÉVÊQUE et GABORIT (1972) qui utilisèrent cette méthode pour décrire les affinités entre 7 espèces de Mollusques. Le succès de l'AFC en écologie vient du fait qu'elle permet l'ordination simultanée des espèces et des relevés et la mise en évidence de gradient (HILL, 1974). Le caractère optimal des codes numériques issus de cette analyse a par ailleurs largement été explicité (WILLIAMS, 1952 ; BENZÉCRI, 1973 ; GREENACRE, 1984). Des applications diverses telles que la reconstitution de classes de taille dans des populations de poissons ou d'invertébrés (PERSAT et CHESSEL, 1989) ou la représentation conjointe de l'amplitude de niche écologique d'espèces et de leur diversité d'habitat (THIOULOUSE et CHESSEL, 1992) sont autant d'exemples montrant la plasticité des modèles utilisables en AFC.

En milieu fluvial, l'échantillonnage ponctuel d'abondance par pêche électrique est utilisée sur le Haut-Rhône français depuis une quinzaine d'années pour obtenir une image représentative des peuplements piscicoles (NELVA et al., 1979 ; PERSAT et al., 1985 ; PERSAT, 1988). L'intérêt reconnu d'une telle méthode de prélèvement dans l'étude des communautés aquatiques associées aux grands fleuves (PERSAT et COPP, 1988) complique cependant le modèle classique de la table de contingence analysé en AFC. En effet, au tableau de contingence (campagnes de pêches-espèces de poissons) est adjoint un effort d'échantillonnage. Cet effort d'échantillonnage correspond au nombre de points de pêche réalisés à une campagne donnée. En d'autres termes, chaque valeur du tableau relevés-espèces correspond en fait à la somme d'un ensemble de relevés. Le problème général posé par cette situation expérimentale renvoie à la notion de profils écologiques (DAGET et GODRON, 1982).

L'objet de l'article est de montrer comment la prise en compte de cet effort d'échantillonnage dans une Analyse Factorielle des Correspondances Décentrée (AFCD) rend compte des modifications d'une structure piscicole non décelée par l'AFC classique. Le développement d'une telle méthode fait appel au schéma de dualité introduit en écologie par ESCOUFIER (1987) et illustré par exemple dans DOLÉDEC et CHESSEL (1991).

\section{MATÉRIEL ET MÉTHODES}

\section{Données}

L'exemple est extrait d'une base de données ichtyologiques mise en oeuvre à partir des échantillons ponctuels d'abondance collectés sur le Haut-Rhône français (ROUSSEAU et al., 1985 ; PERSAT, 1988). Les données traitées sont composées de la distribution de huit espèces de poissons (sept espèces appartenant à la famille des Cyprinidés et une espèce appartenant à la famille des Percidés) recensées au cours de 32 campagnes de pêche, soit plus de 6500 échantillons ponctuels réalisés sur le Rhône en amont de Lyon de 1978 à 1987 (tableau I).

Les analyses décrites ci-après portent sur les profils à long terme de ces espèces de poissons (Fig. 1).

\section{Principe de la méthode}

Soit $\mathbf{N}$ un tableau faunistique comportant / relevés (lignes) et $J$ espèces (colonnes). Chaque relevé $i(1 \leq i<I)$ correspond à une campagne d'échantillonnage comportant $r_{i}$ relevés ponctuels. Le tableau $\mathbf{N}$ comporte à la ligne $i$ et la colonne $j$ la valeur $n_{i j}$ qui est le nombre de relevés de la campagne $i(1 \leq i<\eta)$ contenant l'espèce $j(1 \leq j<J)$. La fréquence relative (notée $f_{j / j}$ ) de l'espèce $j$ (DAGET et GODRON, 1982, p. 30) ou fréquence conditionnelle de l'espèce $j$ dans la campagne i est égale à : 
Tableau I : Exemple de données piscicoles extraites de la base de données décrite par PERSAT (1988). A la ligne $i$ et à la colonne $j$ du tableau, on trouve la valeur $n_{i j}$ qui est le nombre d'échantillons ponctuels d'abondance de la campagne $i(1 \leq i<32)$ où est enregistrée la présence de l'espèce $j(1 \leq j<8)$. Le total marginal en ligne représente pour une campagne de pêche le nombre d'échantillons ponctuels d'abondance où des poissons (toutes espèces confondues) ont été recensés. Le total en colonne représente le nombre d'échantillons ponctuels d'abondance où l'espèce en question a été trouvée. La colonne «r» représente l'effort d'échantillonnage, c'est-àdire le nombre d'échantillons ponctuels d'abondance effectués à chaque campagne de pêche.

Codes des espèces : HOT = Hotu Chondrostoma nasus (L. 1758) ; VAN = Vandoise Leuciscus leuciscus (L. 1758) ; CHE = Chevaine Leuciscus cephalus (L. 1758) ; SPI = Spirlin Alburnoïdes bipunctatus (Bloch 1782) ; GOU = Goujon Gobio gobio (L. 1758) ; BAF = Barbeau fluviatile Barbus barbus (L. 1758) ; GAR = Gardon Rutilus rutilus (L. 1758) ; PER = Perche fluviatile Perca fluviatilis (L. 1758).

Table I : Data example extracted from the fish species data base promoted by PERSAT (1988). The value $n_{i j}$ at the intersection of the ith row and the $j$ th column corresponds to the number of sampling points of the ith sampling occasion $(1 \leq i<32)$ that contains the $j$ th species $(1 \leq j<8)$. Row totals represent the number of sampling points that contain fish species. Column totals represent the number of sampling points that contain a given fish species. The column noted " $r$ " represents the sampling effort, i.e. the total number of sampling points made at each sampling occasion.

Species codes $:$ HOT = Nase Chondrostoma nasus (L. 1758); VAN = Dace Leuciscus leuciscus (L. 1758) ; CHE = Chub Leuciscus cephalus (L. 1758) ; SPI = Streambleak Alburnoïdes bipunctatus (Bloch 1782); GOU = Gudjeon Gobio gobio (L. 1758) ; BAF = Barbel Barbus barbus (L. 1758) ; GAR = Roach Rutilus rutilus (L. 1758) ; PER = Perch Perca fluviatilis (L. 1758).

\begin{tabular}{|c|c|c|c|c|c|c|c|c|c|c|}
\hline Année & HOT & VAN & QEE & $\mathbf{S P I}$ & GOU & BAF & GAA & PER & Total & $\mathbf{r}$ \\
\hline 1978 & 57 & 47 & 60 & 11 & 27 & 17 & 10 & 21 & 250 & 202 \\
\hline 1978 & 9 & 25 & 19 & 6 & 2 & 5 & 0 & 6 & 72 & 57 \\
\hline 1978 & 48 & 60 & 52 & 16 & 25 & 24 & 17 & 12 & 254 & 246 \\
\hline 1979 & 26 & 50 & 32 & 8 & 6 & 11 & 2 & 5 & 140 & 165 \\
\hline 1979 & 26 & 43 & 31 & 14 & 9 & 18 & 13 & 2 & 156 & 203 \\
\hline 1980 & 62 & 43 & 47 & 16 & 11 & 9 & 15 & 3 & 206 & 279 \\
\hline 1981 & 17 & 34 & 28 & 13 & 8 & 13 & 14 & 4 & 131 & 142 \\
\hline 1981 & 18 & 22 & 18 & 9 & 3 & 7 & 6 & 4 & 87 & 110 \\
\hline 1981 & 9 & 14 & 8 & 5 & 8 & 8 & 1 & 0 & 53 & 60 \\
\hline 1981 & 12 & 9 & 9 & 10 & 2 & 2 & 10 & 0 & 54 & 90 \\
\hline 1982 & 23 & 11 & 16 & 18 & 4 & 3 & 8 & 3 & 86 & 161 \\
\hline 1982 & 3 & 1 & 2 & 1 & 2 & 1 & 3 & 0 & 13 & 40 \\
\hline 1982 & 24 & 42 & 26 & 18 & 13 & 20 & 7 & 2 & 152 & 140 \\
\hline 1982 & 22 & 25 & 14 & 14 & 4 & 10 & 17 & 5 & 111 & 131 \\
\hline 1982 & 22 & 18 & 30 & 11 & 11 & 13 & 27 & 10 & 142 & 183 \\
\hline 1982 & 6 & 5 & 6 & 0 & 5 & 2 & 4 & 2 & 30 & 60 \\
\hline 1983 & 43 & 11 & 42 & 13 & 17 & 16 & 64 & 47 & 253 & 300 \\
\hline 1983 & 12 & 8 & 15 & 7 & 9 & 8 & 26 & 11 & 96 & 110 \\
\hline 1983 & 66 & 59 & 67 & 28 & 29 & 64 & 45 & 72 & 430 & 350 \\
\hline 1983 & 24 & 50 & 96 & 24 & 50 & 33 & 50 & 44 & 371 & 350 \\
\hline 1984 & 28 & 25 & 82 & 33 & 63 & 12 & 48 & 53 & 344 & 350 \\
\hline 1984 & 40 & 43 & 75 & 21 & 38 & 28 & 32 & 33 & 310 & 350 \\
\hline 1984 & 50 & 64 & 126 & 40 & 123 & 73 & 91 & 23 & 590 & 350 \\
\hline 1985 & 28 & 21 & 99 & 38 & 102 & 21 & 95 & 25 & 429 & 350 \\
\hline 1985 & 55 & 65 & 106 & 29 & 82 & 46 & 65 & 26 & 474 & 355 \\
\hline 1985 & 64 & 58 & 141 & 34 & 68 & 63 & 63 & 50 & 541 & 275 \\
\hline 1986 & 19 & 20 & 99 & 17 & 17 & 6 & 28 & 30 & 236 & 275 \\
\hline 1986 & 46 & 44 & 108 & 10 & 37 & 48 & 37 & 42 & 372 & 275 \\
\hline 1986 & 25 & 22 & 94 & 20 & 54 & 33 & 62 & 23 & 333 & 150 \\
\hline 1987 & 9 & 7 & 45 & 1 & 38 & 6 & 23 & 11 & 140 & 150 \\
\hline 1987 & 9 & 26 & 40 & 1 & 31 & 10 & 25 & 17 & 159 & 150 \\
\hline 1987 & 34 & 23 & 108 & 5 & 53 & 28 & 99 & 39 & 389 & 150 \\
\hline Total & 936 & 995 & 1741 & 491 & 951 & 658 & 1007 & 625 & 7404 & 6559 \\
\hline
\end{tabular}



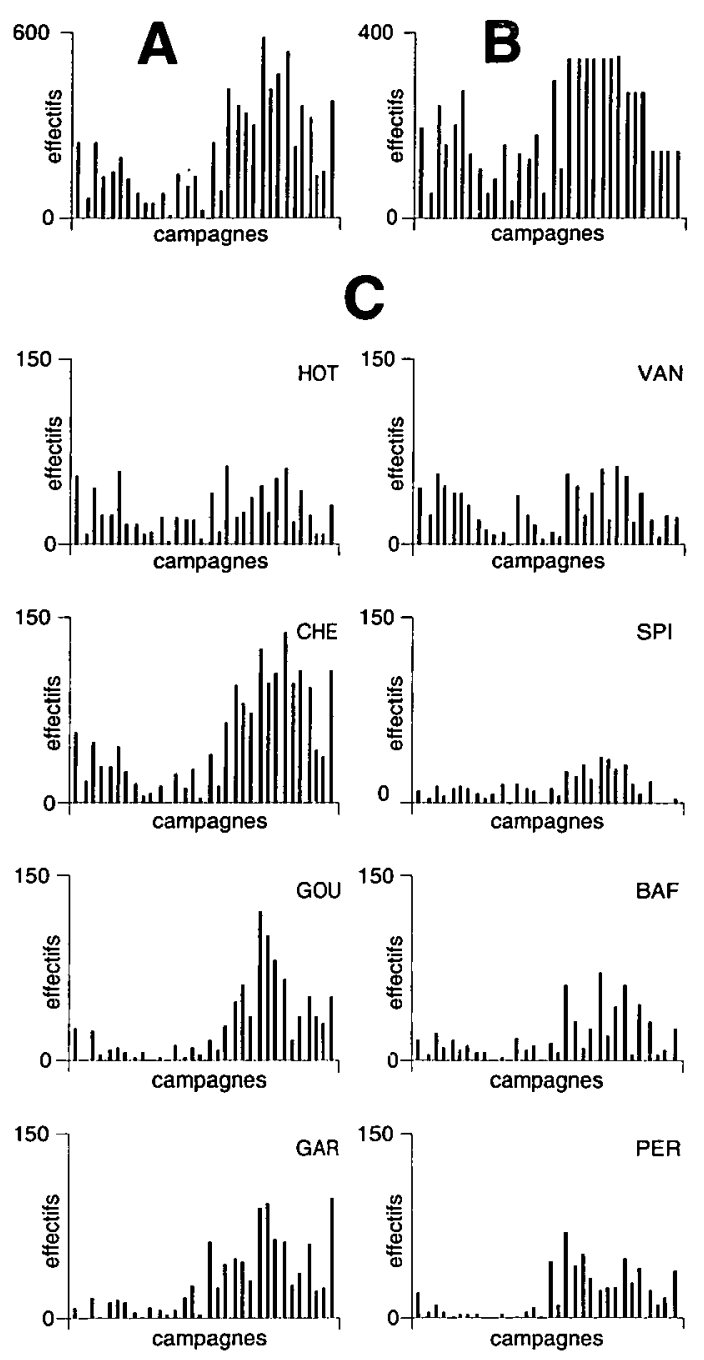

Figure 1 : (A) Nombre total d'échantillons ponctuels d'abondance dans lesquels des espèces piscicoles ont été contactées. (B) Nombre total d'échantillons ponctuels d'abondance par campagne de pêche. (C) Profils d'abondance à long terme de huit espèces piscicoles du Haut-Rhône français recensées au cours de 32 campagnes de pêche de 1978 à 1987 .

Codes des espèces : HOT = Hotu Chondrostoma nasus (L. 1758) ; VAN = Vandoise Leuciscus leuciscus (L. 1758) ; CHE = Chevaine Leuciscus cephalus (L. 1758) ; SPI = Spirlin Alburnoïdes bipunctatus (Bloch 1782) ; GOU = Goujon Gobio gobio (L. 1758) ; BAF = Barbeau fluviatile Barbus barbus (L. 1758) ; GAR = Gardon Rutilus rutilus (L. 1758) ; PER = Perche fluviatile Perca fluviatilis (L. 1758).

Figure 1 : (A) Total number of sampling points presenting species. (B) Total number of sampling points at each sampling occasion. (C) Long-term evolution of the abundance of eight fish species collected at 32 occasions on the French Upper Rhône River from 1978 to 1987.

Species codes $:$ HOT $=$ Nase Chondrostoma nasus $($ L. 1758) ; VAN = Dace Leuciscus leuciscus (L. 1758) ; CHE = Chub Leuciscus cephalus (L. 1758); SPI = Streambleak Alburnoïdes bipunctatus (Bloch 1782); GOU = Gudjeon Gobio gobio (L. 1758) ; BAF = Barbel Barbus barbus (L. 1758) ; GAR = Roach Rutilus rutilus (L. 1758) ; PER = Perch Perca fluviatilis (L. 1758). 


$$
f_{j / i}=\frac{n_{i j}}{r_{i}}
$$

La fréquence totale (notée $f_{j}$ ) de l'espèce $j$ est égale à :

$$
f_{j}=\frac{n_{\cdot j}}{R}
$$

avec $n_{. j}=\sum_{j=1}^{J} n_{i j} \quad$ représentant le nombre de relevés ponctuels contenant l'espèce $j$

et $R=\sum_{i=1}^{l} n_{i j}$ représentant le nombre total de relevés ponctuels $\left(n_{i j} \leq R\right)$. est égale à :

La fréquence corrigée (notée $\frac{f_{j / i}}{f_{j}}$ ) de l'espèce (DAGET et GODRON, 1982, p. 31)
gale à :

$$
\frac{f_{j i}}{t_{j}}=\frac{\frac{n_{i j}}{r_{i}}}{\frac{n_{. j}}{R}}=\frac{\frac{n_{i j}}{n_{j}}}{\frac{r_{i}}{R}}
$$

En d'autres termes, la fréquence corrigée de l'espèce $j$ dans la campagne $i$ est aussi le rapport de la proportion de points présentant l'espèce $j$ à la campagne $i$ et de la proportion de points effectués à la campagne $i$.

II résulte de ces expressions qu'une bonne méthode d'ordination devrait faire référence au vecteur $\left(r_{1}, \ldots, r_{i}, \ldots, r_{i}\right)$ représentant l'effort d'échantillonnage, ce qui n'est pas le cas de l'AFC classique.

Dans la théorie des profils écologiques, les $r_{j}$ sont des réalisations de variables aléatoires (observations a posteriori). Par contre, dans la situation expérimentale proposée, les $r_{i}$ sont des nombres fixés par l'expérimentateur. En conséquence, pour tester la distribution $n_{i j}$ (avec pour marge $n_{j}$ ) d'une espèce, il faut la comparer à la distribution des $r_{i}$ (avec pour marge $R$ ). Sous l'hypothèse nulle que la fréquence de l'espèce est indépendante de la campagne de pêche, on calcule le $\aleph_{j}^{2}$, qui suit une distribution $\aleph^{2}$ à $l-1$ degrés de liberté conformément au test multinomial d'ajustement décrit dans LEBART et al. (1982, p. 134) :

$$
\kappa_{j}^{2}=\sum_{i=1}^{l} \frac{\left(n_{i j}-r_{i} \frac{n_{. j}}{R}\right)^{2}}{r_{i} \frac{n_{. j}}{R}}
$$

Cette expression se développe et peut s'écrire :

$$
\frac{1}{R} N_{j}^{2}=\sum_{i=1}^{l} \frac{r_{i}}{R} f_{j}\left(\frac{f_{j / i}}{f_{j}}-1\right)^{2}
$$


En reprenant le formalisme du schéma de dualité (CAILLIEZ et PAGES, 1976) introduit en écologie par ESCOUFIER (1987), on appelle Analyse Factorielle des Correspondances Décentrée (AFCD), l'analyse du triplet $\left(\mathbf{X}, \mathbf{D}_{\omega}, \mathbf{D}_{n}\right)$ où $\mathbf{X}$ est un tableau à $I$ relevés (lignes) et $j$ espèces (colonnes) de terme général $x_{i j}$ :

$$
x_{i j}=\frac{f_{j / i}}{f_{j}}-1
$$

avec $\frac{f_{j / i}}{f_{j}}$ représentant la fréquence corrigée de l'espèce $j$ (cf. équation 3 ).

$\mathbf{D}_{\omega}=\operatorname{Diag}\left(f_{1}, \ldots, f_{j}, \ldots, f_{j}\right)$ représente le poids des espèces (matrice diagonale).

$\mathbf{D}_{n}=\operatorname{Diag}\left(\frac{r_{1}}{R}, \ldots, \frac{r_{i}}{R}, \ldots, \frac{r_{l}}{R}\right)$ est la diagonale des poids des classes-relevés (campagnes de pêche).

L'inertie totale $I_{T}$ de cette analyse vaut :

$$
I_{T}=\sum_{i=1}^{I} \frac{r_{i}}{R} \sum_{j=1}^{J} f_{j}\left(\frac{f_{j i}}{f_{j}}-1\right)^{2}=\frac{1}{R} \sum_{j=1}^{J} \boldsymbol{N}_{j}^{2}
$$

et cela renvoie à l'équation (5).

Les coordonnées factorielles des lignes (relevés) issues de l'analyse présentent des propriétés remarquables. Elles sont centrées pour la pondération externe $\left(\frac{r_{1}}{R}, \ldots, \frac{r l}{R}\right)$. Elles sont de variances $\lambda_{k}$ (kième valeur propre de l'analyse) et non corrélées pour la pondération $\mathbf{D}_{\omega}$ des colonnes (espèces).

Les espèces (coordonnées factorielles des colonnes) sont positionnées (à un coefficient $1 / \sqrt{\lambda k}$ prêt) à la moyenne des campagnes pour leur distribution de fréquences
de points.

Soit $\mathbf{L}_{k}(i)$ la position des campagnes, la position de l'espèce $j$ est donnée par $\overline{\mathbf{L}_{k / j}}=\sum \frac{n_{i j}}{n_{. j}} \mathbf{L}_{k}(i)$ sous la contrainte que $\sum f_{j}{\overline{L_{k / j}}}^{2}$ soit maximale. L'espèce uniformément répartie est donc positionnée à l'origine par $\sum \frac{r_{i}}{R} L_{K}(I)=0$.

Par conséquent, l'AFCD positionne les campagnes de relevés pour maximiser la moyenne pondérée des carrés des écarts entre la position occupée par une espèce donnée et la position de l'espèce uniformément répartie.

\section{RÉSULTATS}

L'examen des profils d'abondance des huit espèces de poissons permet de déceler des tendances à long terme (cf. Fig. 1). Par exemple, une tendance à l'augmentation des effectifs pour le Chevaine, le Goujon ou le Gardon.

Le problème est alors de dégager au sein de ces profils à long terme une structure qui reflète l'évolution du peuplement ichtyologique à long terme, en particulier en terme d'abondance relative (fréquence), ce qui relève d'un problème d'AFC. 


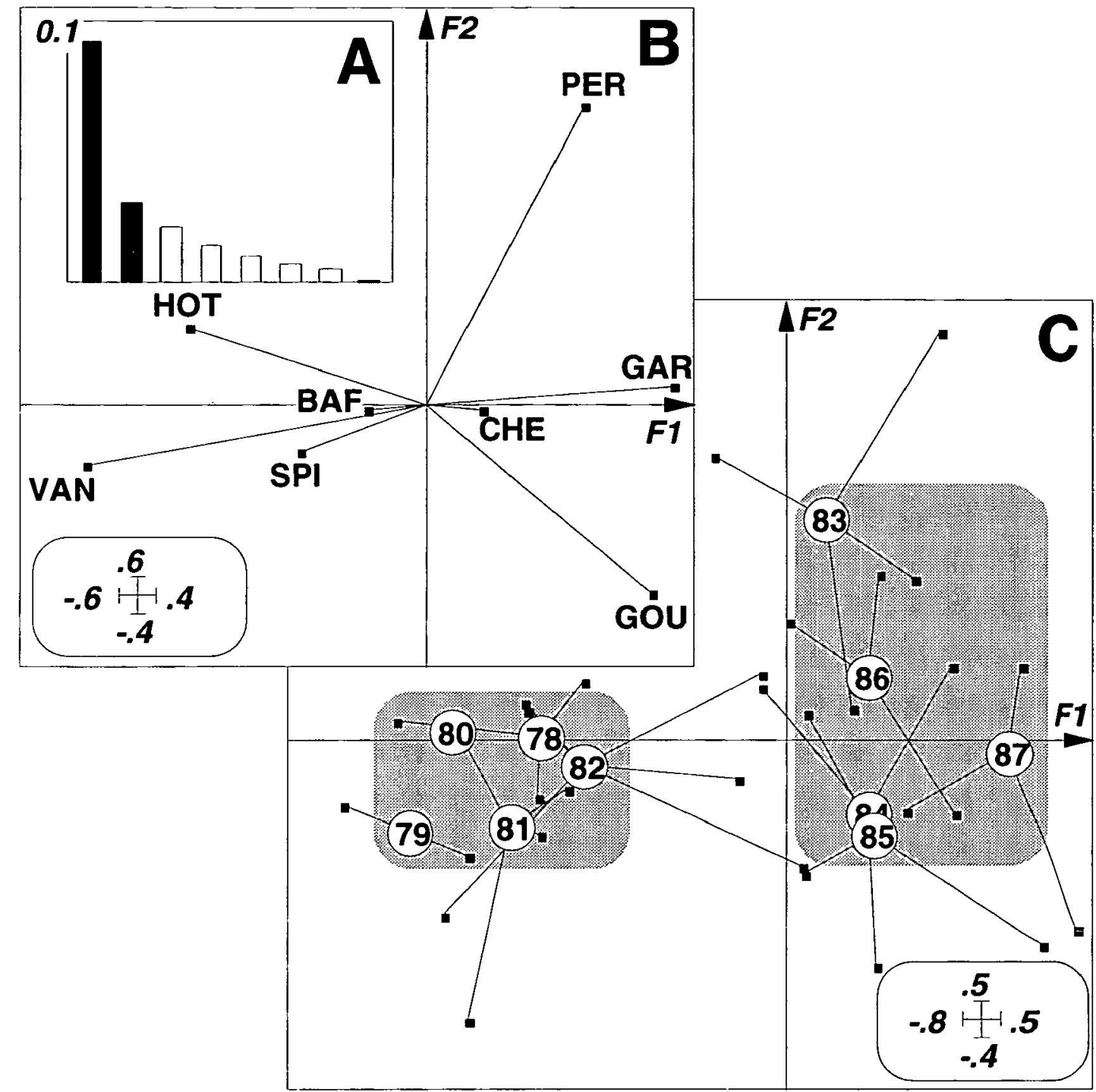

Figure 2 : Résultats de l'AFC du tableau relevés-espèces (cf. tableau 1). (A) Diagramme des valeurs propres identifiant deux axes de structure faunistique (rectangles noirs). (B) Carte F1xF2 des espèces positionnant celles-ci par rapport au profil moyen d'abondance (voir Fig. 1 pour le code des espèces). (C) Carte F1xF2 des relevés. Chaque campagne (carrés) est reliée à l'année correspondante (cercle positionné au centre de gravité des campagnes).

Figure 2 : Results of the correspondence analysis processed on the sample-byspecies matrix (cf. table I). (A) Eigenvalue diagram identifying the two first axes (black bars). (B) Positions of species on the F1xF2 plane. Species are separated by their distance from the average abundance profile. Chub (many individuals) and Barbel (few individuals) positions underscore the ambiguity associated to the reference point implicitly selected in a classical correspondence analysis (see Fig. 1 for species code). (C) Positions of sampling occasions on the F1xF2 plane. Each sampling occasion (small square) is linked to its center of gravity (figures in a circle) situated at the weighted average of the sampling occasions made for a given year. 
L'exemple a donc été successivement traité par l'Analyse Factorielle des Correspondances (AFC) et par l'Analyse Factorielle des Correspondances Décentrées (AFCD). La structure faunistique en oeuvre sur le plan factoriel F1xF2 de l'AFC classique (Fig. 2) sépare un peuplement piscicole présentant des espèces rhéophiles de grande taille (Vandoise, Hotu, Barbeau), d'un peuplement à dominante lénitophile (Gardon, Perche) (Fig. 2B). Cette structure est associée à une séparation en deux groupes de campagnes de pêche (en grisé sur la Fig. 2C). En première approche, cette rupture pourrait être interprétée comme une modification faunistique liée aux aménagements hydroélectriques dont la mise en eau est postérieure à 1981-1982. Cependant, la position du Chevaine sur le plan F1xF2 de l'AFC souligne le poids pris par son profil faunistique (cf. Fig. 1). Les positions du Chevaine (très abondant) et du Barbeau (moins abondant) soulèvent ainsi le problème de la définition du point de référence associé à l'AFC.

La structure faunistique issue de l'AFCD (figure 3 ) est principalement décrite par deux axes (Fig. 3A). Ces axes prennent en compte les variations concomitantes d'abondance des espèces. Ceci est matérialisé par un effet taille, c'est-à-dire que la majorité des espèces présentent des coordonnées de même signe (positives sur la Fig. 3B) sur l'axe F1. La longueur des vecteurs souligne l'éloignement des espèces par rapport à la répartition uniforme et non plus par rapport à l'espèce la plus abondante comme dans l'AFC classique. La direction des vecteurs doit être interprétée à l'aide de la carte des relevés (Fig. $3 \mathrm{C}$ ). La structure faunistique révélée par l'AFCD reprend ainsi à son compte le passage d'un peuplement dominé par la Vandoise (Fig. 3B) au début des pêches (1978, cf. Fig. 3C) à un peuplement dominé par le Gardon à la fin des campagnes (1987, cf. Fig. $3 C$ ), déjà décrit par l'AFC classique.

On peut ainsi suivre (Fig. 3) au fil des campagnes une diminution progressive dans l'abondance de la Vandoise et du Hotu, parallèlement à une augmentation d'abondance du Gardon, de la Perche et du Goujon. La position du Chevaine dont le profil faunistique montre une augmentation d'abondance avec la mise en eau des aménagements hydroélectriques est cette fois clairement prise en compte.

L'examen des corrélations entre l'axe F1 de l'AFCD et l'axe F1 de l'AFC et la corrélation entre l'axe F2 de l'AFCD et l'axe F1 de l'AFC (Fig. 4) soulignent comment l'AFCD décompose les modifications faunistiques observées sur le long terme en un aspect quantitatif et un aspect qualitatif. L'aspect quantitatif pris en compte par l'axe 1 correspond aux variations d'abondance des espèces. L'aspect qualitatif pris en compte par l'axe 2 correspond à une modification de la structure spécifique. L'AFCD enrichit donc l'interprétation alors que seule l'opposition Vandoise-Gardon était prise en compte par l'AFC.

\section{CONCLUSION}

L'AFCD montre des potentialités réelles lorsqu'il s'agit d'étudier de grands volumes d'informations provenant notamment de base de données. L'AFC classique suscite une certaine ambiguité d'interprétation au sens où l'espèce la plus abondante est prise comme espèce de référence. Contrairement à l'AFC, pour laquelle l'élément de référence dépend donc du contenu du tableau étudié, l'AFCD définit le point de référence a priori en prenant en compte la situation expérimentale des objets étudiés. Ces résultats sont des conséquences des théorèmes généraux sur le schéma de dualité revisité par ESCOUFIER (1987). L'AFCD doit être considérée comme un cas particulier de la famille des AFC sur modèles (ESCOUFIER, 1985) qui n'a pas, à notre connaissance, été introduite en écologie. L'AFCD est une solution au problème abọdé dans une étude sociologique par LEBART (1979) qui propose d'ajouter au tableau une ligne ou une colonne supplémentaire pour intégrer l'information extérieure.

La procédure d'intégration du plan d'expérimentation dans une analyse renvoie aussi aux analyses interclasses et intraclasses (DOLÉDEC et CHESSEL, 1991) dont la mise en oeuvre, par exemple, par DEGIORGI et GRANDMOTTET (1993) montre une grande efficacité pour décrire l'organisation spatiale d'une ichtyofaune lacustre.

L'AFCD est disponible dans le logiciel ADE à partir de la version 3.6 (CHESSEL et DOLÉDEC, 1993). 


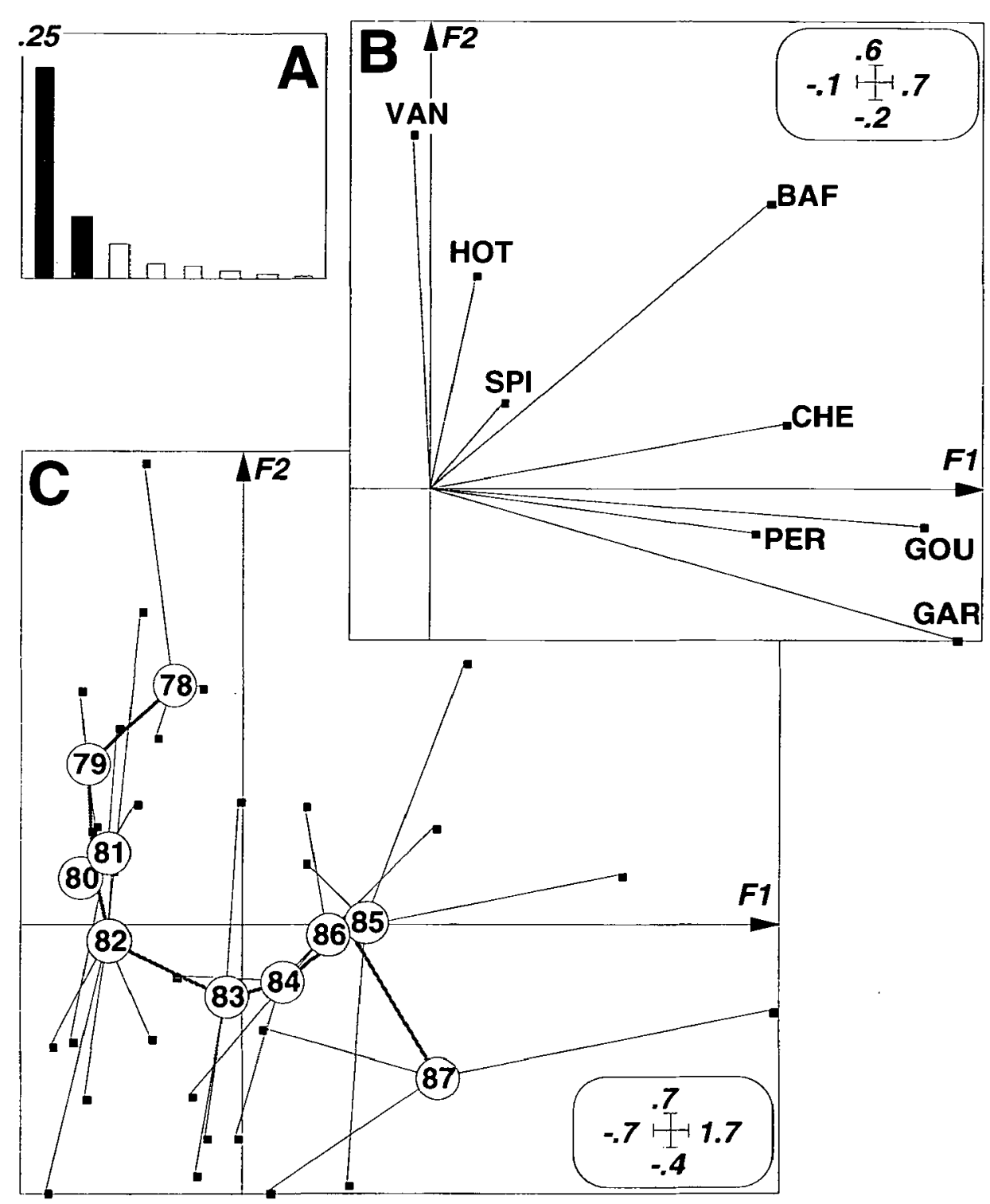

Figure 3 : Résultats de I'AFCD réalisée sur le même tableau relevés-espèces (cf. tableau I). (A) Diagramme des valeurs propres identifiant deux axes de structure faunistique (rectangles noirs). (B) Carte F1xF2 des espèces (voir Fig. 1 pour le code des espèces) positionnant celles-ci par rapport à l'espèce uniformément répartie parmi toutes les campagnes (référence). (C) Carte F1xF2 des relevés où sont positionnées les campagnes de prélèvements (carrés) et leur centre de gravité (cercle).

Figure 3 : Results of the decentred correspondence analysis processed on the same sample-by-species matrix (cf. table I). (A) Eigenvalue diagram identifying the two first axes (black bars). (B) Positions of species (see Fig. 1 for species code) on the F1xF2 plane. Species are separated by their distance from a species uniformely distributed among sampling occasions (reference species). (C) Positions of sampling occasions on the F1xF2 plane. Each sampling occasion (small square) is linked to its center of gravity (figures in a circle) situated at the weighted average of the sampling occasions made for a given year. 

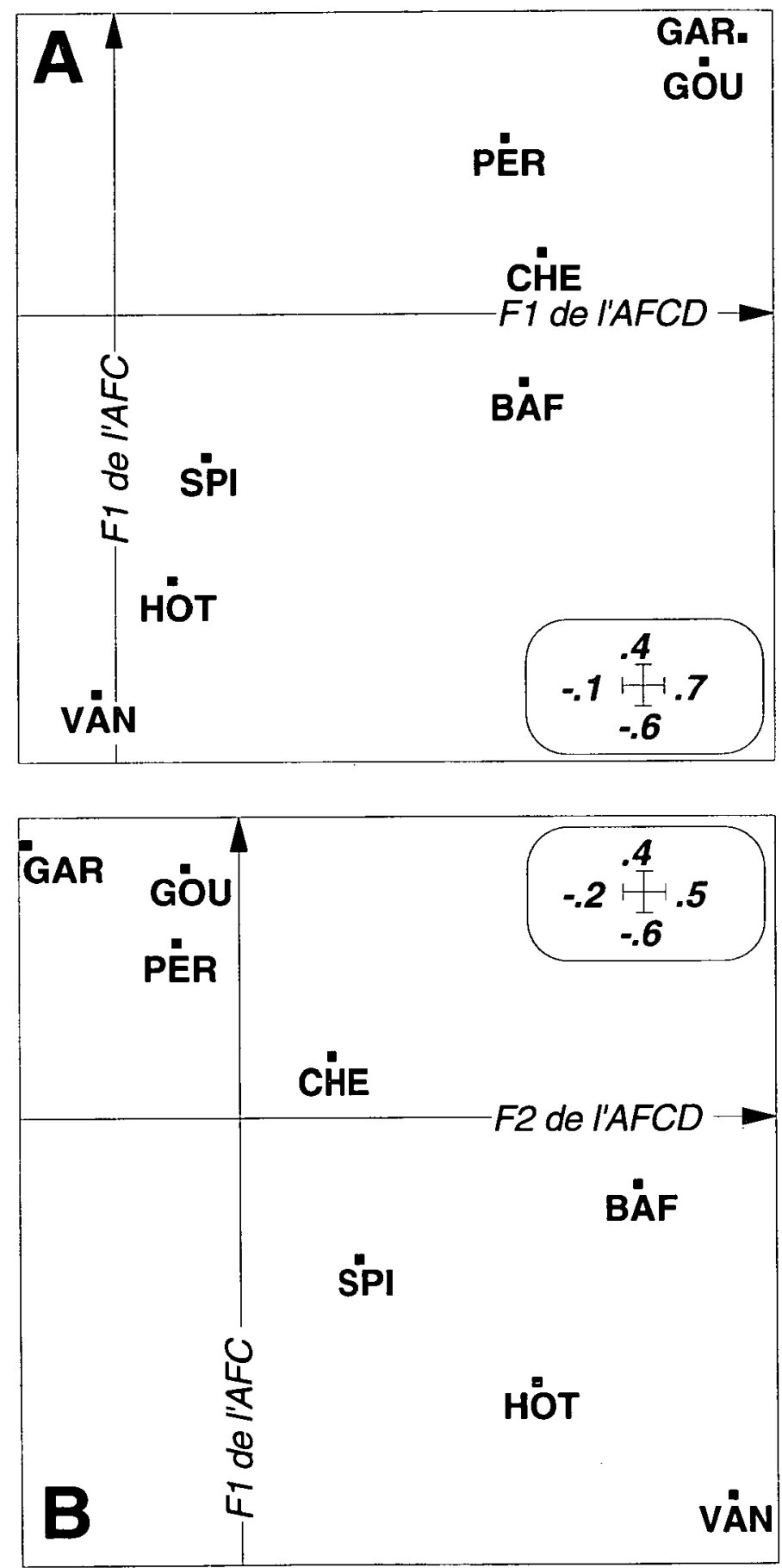

Figure 4 : (A) Corrélation entre l'axe 1 (noté F1) de l'AFCD et l'axe 1 (noté F1) de I'AFC classique. (B) Corrélation entre l'axe 1 de l'AFC (noté F1) et l'axe 2 de l'AFCD (noté F2).

Figure 4 : (A) Bivariate plot using the axis 1 (noted F1) of the correspondence analysis and the axis 1 (noted $F 1$ ) of the decentred correspondence analysis. (B) Bivariate plot using the axis 1 (noted F1) of the correspondence analysis and the axis 2 (noted F2) of the decentred correspondence analysis. 


\section{REMERCIEMENTS}

Nous remercions un lecteur anonyme pour ses suggestions bibliographiques. Les données piscicoles analysées dans ce manuscrit ont été obtenues dans le cadre du suivi hydrobiologique et piscicole des aménagements hydroélectriques du Haut-Rhône, mené par l'Association Rhône-Alpes de Laboratoires pour l'Etude des Problèmes Biologiques de la Pêche que nous remercions.

\section{BIBLIOGRAPHIE}

BENZÉCRI J.P., 1973. L'analyse des données. 2) L'analyse des correspondances. Dunod, Paris, $620 \mathrm{p}$.

CAILLIEZ F., PAGES J.P., 1976. Introduction à l'analyse des données. SMASH, Paris, 616 p.

CHESSEL D., DOLÉDEC S., 1993. ADE Software. Multivariate analyses and graphical display for environmental data. Version 3.6. HyperCard stacks and program library for the analysis of environmental data. Documentation, 8 fascicules, $750 \mathrm{p}$.

DAGET P., GODRON M., 1982. Analyse fréquentielle de l'écologie des espèces dans les communautés. Masson, Paris, $163 \mathrm{p}$.

DEGIORGI F., GRANDMOTTET J.P., 1993. Relations entre la topographie aquatique et l'organisation spatiale de l'ichtyofaune lacustre : définition des modalités spatiales d'une stratégie de prélèvement reproductible. Bull. Fr. Pêche Piscic., 329, 199-220.

DOLÉDEC S., CHESSEL D., 1991. Recent developments in linear ordination methods for environmental sciences. Advances in Ecology, 1, 133-155.

ESCOUFIER Y., 1985. L'analyse des correspondances : ses propriétés et ses extensions. Proceedings of the 45th session of the "Institut International de la Statistique», Amsterdam, 28 (2),1-16.

ESCOUFIER Y., 1987. The duality diagram : a means of better practical applications. In Legendre P. and Legendre L., Development in numerical ecology, 139-156, Springer Verlag, Berlin.

GREENACRE H.J., 1984. Theory and applications of correspondence analysis. Academic Press, Londres, $364 \mathrm{p}$.

HILL M.O., 1974. Correspondence analysis : a neglected multivariate method. Journal of the Royal Statistical Society (London), 23, 340-354.

LEBART L., 1979. Exemple d'analyse d'un tableau dont l'une des colonnes a un poids prédominant. Les Cahiers de l'Analyse des Données, 4, 417-422.

LEBART L., MORINEAU A., FÉNELON J.P., 1982. Traitement des données statistiques. Méthodes et Programmes, Dunod, Paris, $518 \mathrm{p}$.

LÉVÊQUE C., GABORIT M., 1972. Utilisation de l'analyse factorielle des correspondances pour l'étude des peuplements en mollusques benthiques du lac Tchad. Cahiers ORSTOM Série Hydrobiologie, 6, 47-66.

NELVA A., PERSAT H., CHESSEL D., 1979. Une nouvelle méthode d'étude des peuplements ichtyologiques dans les grands cours d'eau par échantillonnage ponctuel d'abondance. C.R. Acad. Sci. Paris, 289, Série D, 1295-1298.

PERSAT H., 1988. De la biologie des populations de l'ombre commun Thymallus thymallus (L. 1758) à la dynamique des communautés dans un hydrosystème fluvial aménagé. Thèse d'état, Univ. Lyon 1, $223 \mathrm{p}$.

PERSAT H., NELVA A., CHESSEL D., 1985. Approche par l'analyse discriminante sur variables qualitatives d'un milieu lotique, le Haut-Rhône français. Acta Oecologica, Oecol. gener., 6, 365-381. 
PERSAT H., COPP G.H., 1988. Electrofishing and point abundance sampling for ichtyology of large rivers. In Cowx I.G., Development in electric fishing, 197-209, Fishing News Books, Cambridge.

PERSAT H., CHESSEL D., 1989. Typologie de distributions en classes de taille : intérêt dans l'étude des populations de poissons et d'invertébrés. Acta Oecologica, Oecol. gener., 10, 175-195.

ROUSSEAU B., NELVA A., PERSAT H., CHESSEL D., 1985. Constitution d'une base de données ichtyologiques par l'échantillonnage ponctuel d'abondance : application aux peuplements du Haut-Rhône français. Cybium, 9, 157-173.

THIOULOUSE J., CHESSEL D., 1992. A method for reciprocal scaling of species tolerance and sample diversity. Ecology, 73, 671-680.

WILLIAMS E.J., 1952. Use of scores for the analysis of association in contingency tables. Biometrika, 39, 274-289. 